\title{
Comment on "nontuberculous mycobacterial infection after lung transplantation: a report of four cases"
}

\author{
Masoud Keikha ${ }^{1,2}$ and Kiarash Ghazvini ${ }^{1,2^{*}}$
}

\begin{abstract}
Nontuberculous mycobacteria can lead to pulmonary infection in lung transplantation cases, which is associated with high mortality rates. We hereby thank Dr. Ose et al. very much for their report on "Nontuberculous mycobacterial infection after lung transplantation: A report of four cases"; however, we have confronted with several questions to have a new review of these cases.
\end{abstract}

Keywords: Nontuberculous mycobacteria (NTM), Infection, Lung, Transplantation, Treatment, Molecular identification

\section{Dear Editor;}

Dr. Ose et al. recently published their report on "Nontuberculous mycobacterial infection after lung transplantation: A report of four cases" [1]. Nontuberculous mycobacteria (NTM) are saprophytes, which are free-living organisms in the surrounding environment such as water, soil, milk, dust, decaying vegetables, and animals [2]. NTM were considered as non-pathogenic mycobacteria until the first report by Prissick and Masson in the 1950s about cervical lymphadenitis caused by chromogenic mycobacteria [3]. There have been numerous evidences in recent years for the isolation of NTM from hospital environment sources, and it is suggested that NTM spp. are transmitted to the hospitalized patients throughout contaminated ventilators, aerosols, catheters, and medical equipment, causing NTM infections in susceptible individuals, including AIDS (acquired immunodeficiency syndrome), cancer, transplantation, or in the immune-compromised patients [4].

According to the American Thoracic Society (ATS) recommendations, NTM infections should be identified to the species level, because of clinical significance, patient management, appropriate treatment, and epidemiological

\footnotetext{
* Correspondence: ghazvinik@mums.ac.ir

${ }^{1}$ Antimicrobial Resistance Research Center, Mashhad University of Medical Sciences, Mashhad, Iran

2Department of Microbiology and Virology, Faculty of Medicine, Mashhad University of Medical Sciences, Mashhad, Iran
}

goals; based on the ATS criteria, NTM pulmonary infection is diagnosed according to a single isolation of NTM spp. from bronchoalveolar lavage (BAL) or pulmonary biopsy, the isolation of NTM spp. in at least two separate sputum specimens, or clinical symptoms with radiographic findings $[5,6]$. According to review of literatures, treatment options for NTM infections are different from Mycobacterium tuberculosis $(M t b)$ infection. In addition, NTM spp. are subdivided into two categories of rapidgrowing mycobacteria (RGM) and slow growing mycobacteria (SGM); unfortunately, RGM are resistant to the therapeutic option of SGM infection. Therefore, identification of NTM to the species level plays a key role in appropriate treatment of NTM infection [7]. However, the emergence of drug-resistant species, particularly $M$. fortuitum, M. chelonae, and M. abscessus, has serious challenges and is associated with a high rate of mortality [8].

Conventional identification of NTM species, considering the factors such as growth rate, pigment production, growth on MacConkey agar, iron uptake, urease production, tween 80 hydrolysis, nitrate reduction, niacin production, catalase, tellurite reduction, aryl sulfatase (3 days), sensitivity to antimicrobial agents, or HPLC (highperformance liquid chromatography) analysis of mycolic acid, is expensive and labor-intensive, needs trained technicians, and takes several weeks for the assessments. However, molecular methods including sequencing, line probe assay, PCR-RFLP (polymerase chain reaction-restriction 
fragment length polymorphism), or LAMP (loop-mediated isothermal amplification) using housekeeping genes of $16 \mathrm{~S}$ rRNA, hsp65, rpoB, secA1, tuf, gyrB, or recA are reliable methods for rapid, inexpensive, and accurate identification of NTM species, particularly in emergence of disseminated infections [9].

There are several case reports of NTM infections in lung transplantation issues; Valentine et al. have shown that Mycobacterium abscessus, Mycobacterium avium complex, Mycobacterium gordonae, Mycobacterium chelonae, and Mycobacterium fortuitum are common NTM species, which are isolated from pulmonary infection of lung transplantation cases besides multiple NTM infections [10]. In addition, it is estimated that $0.46-9 \%$ of lung transplantation were fallen to NTM infection, in relation with the transplant rejection or high mortality rates (even $44 \%$ mortality) $[6,11,12]$. We should thank Dr. Ose et al. very much for this article, and it is presumed that developing these studies can be useful for the appropriate management of NTM infection of transplantation cases. However, we hereby request the authors to pay attention to the following issues:

1. Please describe the mycobacterial isolation method, which was not stated in the report.

In the diagnosis of NTM infection in a lung transplant patient, it can be noticed that the infection can occur by contamination or transient colonization in the conventional method by bronchoalveolar lavage or biopsy and sputum-positive culture. This case did not show a clinical symptom and completely recovered with no chemotherapy, which could be considered as transient colonization

2. Please explain how the Mycobacterium spp. were finally identified to the species level that was not declared in this report.

3. What is the basis of the antibiotic regimen selection, whereas there is no information about performing an antibiotic susceptibility test in this report?

4. Please describe the previous infection of the lung transplantation cases and also give more details about the clinical symptoms of the cases.

5. Please tell us about the role of this bacterium in the lung transplantation and also about the lack of relevance of the lung transplantation infections with other bacteria.

\footnotetext{
Abbreviations

AIDS: Acquired immunodeficiency syndrome; ATS: American Thoracic Society; HPLC: High-performance liquid chromatography; LAMP: Loopmediated isothermal amplification; Mtb: Mycobacterium tuberculosis; Mycobacterium spp.: Mycobacterial species; NTM: Non-tuberculosis mycobacteria; PCR-RFLP: Polymerase chain reaction-restriction length polymorphism; RFLP: Restriction fragment length polymorphism; RGM: Rapidgrowing mycobacteria; SGM: Slow-growing mycobacteria
}

\section{Acknowledgements}

Not applicable.

\section{Authors' contributions}

KG described and designed the article and supervised the edition of the manuscript. MK did the writing of the manuscript. Both authors read and approved the final manuscript.

\section{Funding}

This study was not funded.

\section{Availability of data and materials}

There were no available repositories.

Ethics approval and consent to participate

Not applicable for this study.

Consent for publication

Not applicable.

\section{Competing interests}

The authors declare that they have no competing interests.

Received: 15 April 2019 Accepted: 16 July 2019

Published online: 23 July 2019

References

1. Ose N, Minami M, Funaki S, Kanou T, Kanzaki R, Shintani $Y$, et al. Nontuberculous mycobacterial infection after lung transplantation: a report of four cases. Surg Case Rep. 2019;5(1):11.

2. Keikha M. Comment on Mycobacterium chelonae infection of the buttocks secondary to lipofilling: a case report and review of the literature. Aesthet Surg J. 2018:42(2):610-2.

3. Prissick FH, Masson AM. Cervical lymphadenitis in children caused by chromogenic mycobacteria. Can Med Assoc J. 1956;75(10):798.

4. Van Ingen J, Boeree MJ, Dekhuijzen PN, Van Soolingen D. Environmental sources of rapid growing nontuberculous mycobacteria causing disease in humans. Clin Microbiol Infect. 2009;15(10):888-93.

5. Griffith DE, Aksamit T, Brown-Elliott BA, Catanzaro A, Daley C, Gordin F, et al. An official ATS/IDSA statement: diagnosis, treatment, and prevention of nontuberculous mycobacterial diseases. Am J Respir Crit Care Med. 2007; 175(4):367-416

6. Cohen L, Guarner J, Hunt WR. A novel presentation of Mycobacterium avium complex in a recipient of a lung transplant: a case report. J Med Case Rep. 2017;11(1):240

7. Wagner D, Young LS. Nontuberculous mycobacterial infections: a clinical review. Infect. 2004;32(5):257-70.

8. Brown BA, Wallace RJ, Onyi GO, De Rosas V. Activities of four macrolides, including clarithromycin, against Mycobacterium fortuitum, Mycobacterium chelonae, and M. chelonae-like organisms. Antimicrob Agents Chemother. 1992;36(1):180-4.

9. Hashemi-Shahraki A, Bostanabad SZ, Heidarieh P, Titov LP, Khosravi AD, Sheikhi N, Ghalami M, Nojoumi SA, et al. Species spectrum of nontuberculous mycobacteria isolated from suspected tuberculosis patients, identification by multi locus sequence analysis. Infect Genet Evol. 2013;20:312-24.

10. Shah SK, McAnally KJ, Seoane L, Lombard GA, LaPlace SG, Lick S, Dhillon GS, Valentine VG, et al. Analysis of pulmonary non-tuberculous mycobacterial infections after lung transplantation. Transpl Infect Dis. 2016:18(4):585-91.

11. Keikha M. Comment on report of two cases of cutaneous Mycobacterium abscessus infection complicating professional decorative tattoo. Dermatol Sin. 2019;37(1):56-7.

12. Huang HC, Weigt SS, Derhovanessian A, Palchevskiy V, Ardehali A, Saggar R, Saggar R, Kubak B, Gregson A, Ross DJ, Lynch JP III, et al. Non-tuberculous mycobacterium infection after lung transplantation is associated with increased mortality. J Heart Lung Transplant. 2011;30(7):790-8.

\section{Publisher's Note}

Springer Nature remains neutral with regard to jurisdictional claims in published maps and institutional affiliations. 